\title{
Survivors Versus Nonsurvivors Postburn
}

\section{Differences in Inflammatory and Hypermetabolic Trajectories}

\author{
Marc G. Jeschke, MD, PhD, † Gerd G. Gauglitz, MD, $\S$ Celeste C. Finnerty, PhD, ${ }^{*} \dagger \ddagger$ Robert Kraft, MD, ${ }^{*} \dagger$ \\ Ronald P. Mlcak, PhD, ${ }^{*} \dagger$ and David N. Herndon, $M D^{*} \dagger$
}

\begin{abstract}
Objective: To evaluate whether a panel of common biomedical markers can be utilized as trajectories to determine survival in pediatric burn patients.

Background: Despite major advances in clinical care, of the more than 1 million people burned in the United States each year, more than 4500 die as a result of their burn injuries. The ability to predict patient outcome or anticipate clinical trajectories using plasma protein expression would allow personalization of clinical care to optimize the potential for patient survival. Methods: A total of 230 severely burned children with burns exceeding $30 \%$ of the total body surface, requiring at least 1 surgical procedure were enrolled in this prospective cohort study. Demographics, clinical outcomes, and inflammatory and acute-phase responses (serum cytokines, hormones, and proteins) were determined at admission and at 11 time points for up to 180 days postburn. Statistical analysis was performed using a 1-way analysis of variance, the Student $t$ test, $\chi^{2}$ test, and Mann-Whitney test where appropriate.

Results: Survivors and nonsurvivors exhibited profound differences in critical markers of inflammation and metabolism at each time point. Nonsurvivors had significantly higher serum levels of interleukin (IL)-6, IL-8, granulocyte colony-stimulating factor, monocyte chemoattractant protein-1, C-reactive protein, glucose, insulin, blood urea nitrogen, creatinine, and bilirubin $(P$ $<0.05$ ). Furthermore, nonsurvivors exhibited a vastly increased hypermetabolic response that was associated with increases in organ dysfunction and sepsis when compared with survivors $(P<0.05)$.

Conclusions: Nonsurvivors have different trajectories in inflammatory, metabolic, and acute phase responses allowing differentiation of nonsurvivors from survivors and now possibly allowing novel predictive models to improve and personalize burn outcomes.
\end{abstract}

Keywords: burn size, critical care, hypermetabolism, inflammation, mortality, survival, pediatric

(Ann Surg 2014;259:814-823)

From the * Shriners Hospitals for Children; †Department of Surgery, University of Texas Medical Branch; $\ddagger$ Institute for Translational Science and Sealy Center for Molecular Medicine, University of Texas Medical Branch, Galveston, TX; §Department of Dermatology and Allergy, Ludwig Maximilians University, Munich, Germany; and $\uparrow$ Ross Tilley Burn Centre, Sunnybrook Health Sciences Centre and Sunnybrook Research Institute, Departments of Surgery and Immunology, Division of Plastic Surgery, University of Toronto, Ontario, Canada.

M.G.J., G.G.G., C.C.F. contributed equally to this work.

Disclosure: This study was supported by grants from Shriners Hospitals for Children $(71008,8760,8740,8507,84080$, and 79135), National Institutes of Health (R01 GM56687, R01 GM087285, T32 GM008256, P50 GM60338, H133 A070026, CIHR 123336), CFI Leader's Opportunity Fund: Project (25407), and Physicians' Services Incorporated Foundation-Health Research Grant Program. CCF is an Institute for Translational Sciences Career Development Scholar supported, in part, by NIH (KL2RR029875 and UL1RR029876). The authors declare no conflicts of interest.

Reprints: Marc G. Jeschke, MD, PhD, Ross Tilley Burn Centre, Sunnybrook Health Sciences Centre and Sunnybrook Research Institute, Departments of Surgery and Immunology, Division of Plastic Surgery, University of Toronto, Ontario, Canada, Rm D704, 2075 Bayview Ave, Toronto, ON M4N 3M5, Canada. Email:marc.jeschke@sunnybrook.ca.

Copyright (C) 2013 by Lippincott Williams \& Wilkins

ISSN: 0003-4932/13/25904-0814

DOI: $10.1097 /$ SLA.0b013e31828dfbf1
$\mathrm{T}_{\mathrm{r}}^{\mathrm{ratan}}$ reatment of severely burned patients has dramatically changed over the last decades leading to improved outcomes, so that survival from a severe burn is no longer the exception but the rule, even for victims at the extremes of age. ${ }^{1-3}$ Unfortunately, although severely burned patients are more likely to survive, death still occurs. Known primary determinants of mortality from severe burn injury are age, presence of inhalation injury, sepsis, and burn size. ${ }^{1-3}$ Besides these factors, another major contributor to adverse outcomes is the profound hypermetabolic response. ${ }^{2,4}$ The magnitude and duration of this metabolic response is related to the severity of the original burn injury. ${ }^{5}$ This response significantly retards growth and induces devastating muscle and protein catabolism, insulin resistance, and cardiac dysfunction that persist for months after discharge. ${ }^{4-7}$ Patients have supraphysiologic metabolic rates, develop multiple organ dysfunction, and express increased levels of systemic inflammatory cytokines and acute-phase proteins. ${ }^{4,5}$ More recently, trauma-induced hyperglycemia has been linked to poor outcomes such as impaired wound healing, increased skin graft loss, augmented muscle protein catabolism, and a greater incidence of infection and mortality. 8,9

Despite the large impact of the hypermetabolic, inflammatory, and acute phase responses on patient outcome, the clinicians' ability to predict survival has remained limited. The "criterion standard" of age, burn size, and inhalation injury traditionally has been used to predict mortality. We have recently shown, however, that this set of predictors is only accurate for predicting nonsurvival for $54 \%$ of patients who die. ${ }^{10} \mathrm{We}$ and others have used cytokines and other plasma markers to create biomarker profiles in an attempt to predict patient outcome. ${ }^{10-12}$ However, these studies were marked by limitations such as single time points, small sample numbers, and poorly defined endpoints such as mortality. Hence, to date, no large study exists that prospectively identifies biomarkers and biomarker trajectories associated with mortality. Identification of potential biomarkers would not only enable identification of patients unlikely to survive burn injury, but also allow for individualized treatment, including heightened monitoring of clinical progression and more aggressive treatment to greatly improve outcome. Here, we conducted a prospective cohort study to compare trajectories of common markers of metabolism and inflammation for use as biomarkers of survival in severely burned patients.

\section{MATERIALS AND METHODS}

\section{Patients}

This study was reviewed and approved by the institutional review board of the University of Texas Medical Branch, Galveston, Texas. Before the study, each subject, parent, or child's legal guardian signed a written informed consent form. All thermally injured pediatric patients included in this study had burns covering more than $30 \%$ of their total body surface area (TBSA), were admitted over a 12 -year period, required at least 1 surgical intervention, and consented to an experimental protocol approved by the institutional review board. 


\section{Admission Data}

All patients were treated at the Shriners Hospitals for ChildrenGalveston pediatric burn intensive care unit according to standardized protocols. ${ }^{4}$ Patients were resuscitated if needed, according to the Galveston formula, with $5000 \mathrm{~mL} / \mathrm{m}^{2}$ TBSA burned $+2,000 \mathrm{~mL} / \mathrm{m}^{2}$ TBSA lactated Ringer's solution being given in increments over the first 24 hours. Within 24 hours of admission, all patients underwent total burn wound excision. The wounds were then covered with available autograft skin, and any remaining open areas were covered with homograft. The donor site healed within 5 to 10 days after the first operative procedure. At this time, patients returned to the operation theater. The procedure was then repeated until all open wound areas were covered with autologous skin material. The time between operations served as an index of wound healing/reepithelialization. This time has previously been shown to be indicative of when donor sites are healed and allows for determination of wound healing.

All patients received the same nutritional treatment according to a standardized protocol. Intake was calculated as follows: 1500 $\mathrm{kcal} / \mathrm{m}^{2}$ body surface $+1500 \mathrm{kcal} / \mathrm{m}^{2}$ area burn. Alternatively, intake was determined by measuring the resting energy expenditure (REE) and multiplying this value by 1.4 with weekly adjustments, as previously described. ${ }^{4}$

\section{Patient Data and Clinical Characteristics}

Upon admission, burn size and severity were assessed and recorded on a standard Lund and Browder chart by the attending burn surgeon. Patient demographics (age, date of burn and admission, gender, burn size, and depth of burn) and concomitant injuries such as inhalation injury, sepsis, morbidity, and mortality were recorded. Sepsis was defined as a positive blood culture or pathologic tissue culture identifying the pathogen during hospitalization or at autopsy, in combination with at least 3 of the following: leucocytosis or leucopenia $(>12,000$ or $<4000)$, hyperthermia or hypothermia $(>38.5$ or $<36.5^{\circ} \mathrm{C}$ ), tachycardia ( $>150$ beats per minute in children), refractory hypotension (systolic blood pressure $<90 \mathrm{~mm} \mathrm{Hg}$ ), thrombocytopenia (platelets $<50,000 / \mathrm{mm}^{3}$ ), hyperglycemia (serum glucose $>240 \mathrm{mg} / \mathrm{dL}$ ), and enteral feeding intolerance (residuals $>200 \mathrm{~mL} / \mathrm{h}$ or diarrhea $>1 \mathrm{~L} /$ day) as previously published., ${ }^{4,13}$

\section{Time Points}

Results obtained during the 180-day period after burn injury were divided into 11 different time blocks: 0 to 1,2 to 7,8 to 10 , 11 to 16,17 to 22,23 to 28,29 to 34,35 to 40,41 to 60,61 to 90 , and 91 to 180 days postburn. These time points were chosen according to admission, operations, and recovery periods. These time points resulted from many discussions and previous studies. Multiple measurements were collected from each patient; for each assessment, the average value reported at each time point is the average of 20 to 110 measurements; on the heat map, average values may include 1 to 4 measurements - these cases are indicated by "XXX" within the cell for that time point. These data were included as little information exists for late time points in patients who do not survive the injury. One-hundred seven nonburned children who consented for research studies and required blood and/or 24-hour urine collections served as the normal cohort; these values have been previously reported. ${ }^{4,6,7}$

\section{Serum Cytokine, Protein, and Hormone Measurements}

Blood was collected from burn patients for serum cytokine and hormone analysis per hospital protocol. Collections were performed at the time of admission, preoperatively, every Monday and Thursday at 6:00 AM, and during subsequent clinical visits for surgical or rehabilitation services. Blood was drawn in a serum-separator collection tube and centrifuged for 10 minutes at 1320 revolutions per minute. The resulting serum was removed and stored at $-70^{\circ} \mathrm{C}$ until assayed. Serum hormones were determined using high performance liquid chromatography and enzyme-linked immunosorbent assay techniques. ${ }^{4}$ The Bio-Plex Human Cytokine 17-Plex panel was used with the Bio-Plex Suspension Array System (Bio-Rad, Hercules, CA) to profile expression of interleukin (IL)-1 $\beta$, IL-2, IL-4, IL-5, IL-6, IL-7, IL-8, IL-10, IL-12p70, IL-13, IL-17, granulocyte colony-stimulating factor (G-CSF), granulocyte-macrophage CSF (GM-CSF), interferon-gamma (IFN- $\gamma$ ), monocyte chemoattractant protein-1 (MCP-1), macrophage inflammatory protein-1-beta (MIP$1 \beta$ ), and tumor necrosis factor (TNF) ${ }^{4,14}$ The assay was performed according to the manufacturer's instructions. Levels of acute phase and constitutive proteins were measured using a nephelometric technique (BN II Plasma Protein Analyzer, Dade Behring/Siemens Healthcare, Deerfield, IL) according to manufacturer's instructions. ${ }^{4,7}$

\section{Indirect Calorimetry}

As part of routine clinical practice, all patients underwent REE measurements weekly during acute hospitalization and during admissions for reconstructive operations performed between discharge and 180 days postburn. REE was measured using a Sensor-Medics Vmax 29 metabolic cart (Yorba Linda, CA) as previously published. ${ }^{4}$ REE was calculated from oxygen consumption and carbon dioxide production by equations already described. ${ }^{15}$ For statistical comparison, measured energy expenditure was expressed as the percentage of the basal metabolic rate predicted compared with predicted norms based upon the Harris-Benedict equation and to body mass index. ${ }^{15}$

\section{Cardiac Function}

M-Mode echocardiograms were completed as follows: at the time of the study, none of the patients presented with or previously suffered from other concomitant diseases affecting cardiac function, such as diabetes mellitus, coronary artery disease, long-standing hypertension, or hyperthyroidism. Study variables included resting cardiac output $(\mathrm{CO})$, cardiac index (CI), stroke volume (SV), resting heart rate, and left ventricular ejection fraction. SV and CO were adjusted for body surface area and expressed as indexes. All cardiac ultrasound measurements were made with the Sonosite Titan echocardiogram with a $3.5 \mathrm{MHz}$ transducer. Recordings were performed with the subjects in a supine position and breathing freely. M-Mode tracings were obtained at the level of the tips of the mitral leaflets in the parasternal long axis position, and measurements were performed according to the American Society of Echocardiography recommendations. Left ventricular volumes determined at end diastole and end systole were used to calculate EF, SV, CO, and CI. Three measurements were performed and averaged for data analysis. ${ }^{4,16}$

\section{Ethics and Statistics}

Analysis of variance with post hoc Bonferroni's correction, paired and unpaired Student $t$ tests, $\chi^{2}$ analysis, and Mann-Whitney tests were used where appropriate. Data are expressed as mean \pm SD or SEM, as indicated. Values of $P$ less than 0.05 were accepted as significant. A Kaplan-Meier curve is presented to show mortality.

\section{Role of the Funding Source}

All funding sources had no involvement in data collection, data analysis, data interpretation, and writing of the report. All authors had access to the data, vouch for its accuracy and completeness, and approved the final report before submission for publication. 


\section{RESULTS}

\section{Patient Characteristics}

Two-hundred thirty severely burned children were enrolled in this study; 79 died within 300 days of the burn injury (Fig. 1). The characteristics of the surviving and nonsurviving patients are provided in Table 1. The survivor and nonsurvivor groups had a similar age, burn size, extent of third-degree burns, and time from burn to admission $(P>0.05)$. Nonsurvivors had a significantly higher maximal Denver 2 Score $(6.7 \pm 2.2$ vs $3.4 \pm 1.5, P<0.001)$ as well as higher incidences of multiple organ failure $(79 \%$ vs $27 \%, P<$ $0.001)$ and septic events $(54 \%$ vs $7 \%, P<0.001)$. No differences in the number of infections and the incidence of inhalation injury were detected between groups (Table 1).

\section{Serum Inflammatory and Acute Phase Responses}

Most of the cytokines measured were significantly altered in response to burn injury, which is in agreement with previous

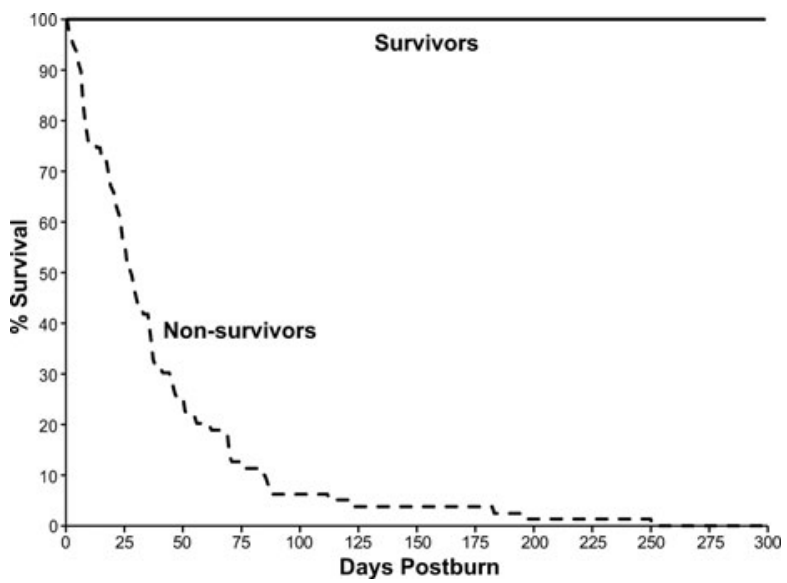

FIGURE 1. Time-dependent changes in survival after burn. studies. ${ }^{4,7,14}$ By comparing severely burned survivors and nonsurvivors, we found significant differences in serum levels of IL- $1 \beta$, IL2, IL-4, IL-5, IL-6, IL-7, IL-8, IL-10, IL-12, IL-17, GM-CSF, INF- $\gamma$, TNF- $\alpha$, G-CSF, MCP-1, and MIP-1 $\beta$ (Fig. 2). Dramatic differences were observed in serum levels of IL-6, IL-8, G-CSF, and MCP-1, $P<0.05$ (Fig. 2). These cytokines increased by up to 10000 -fold in nonsurviving patients and remained significantly elevated throughout the study period when compared with surviving patients. Although IL-6, G-CSF, and MCP-1 rapidly increased after burn, IL-8 could not be used to distinguish between survivors and nonsurvivors until 8 to 10 days postburn. At this point, survivors began to return to normal, whereas in nonsurvivors, IL-8 levels increased and remained elevated for the duration of the study, $P<0.05$. IL-5, IL-10, GM-CSF, INF- $\gamma$, TNF- $\alpha$, and MIP-1 $\beta$ were 2 - to 20 -fold higher in nonsurvivors than in survivors at most time points studied. On the contrary, levels of IL- $1 \beta$, IL-2, IL-4, IL-7, IL-12, and IL-17 were significantly increased at specific time points, with there being no discernible pattern of secretion for these cytokines, $P<0.05$. Serum levels of IL-13 did not significantly differ between groups.

Serum levels of acute-phase proteins were significantly altered upon burn injury, as previously described. ${ }^{4,7}$ Complement $\mathrm{C} 3$, $\alpha_{2}$-macroglobulin, haptoglobin, and $\alpha_{1}$-acid glycoprotein were moderately, albeit significantly, lower in nonsurvivors than in survivors (Fig. 3A-D). In contrast, C-reactive protein levels were significantly higher in the nonsurvivor group (Fig. 3E). Serum levels of the constitutively expressed hepatic protein retinol-binding protein were moderately higher in nonsurvivors than in survivors beginning 2 to 7 days after injury and continuing until 35 to 40 days after injury $(P<$ 0.05 ) (Fig. 3H). The reverse was true for the other constitutively expressed hepatic proteins - prealbumin and transferrin. These proteins were moderately lower in nonsurvivors than in survivors beginning 11 to 16 days postburn and lasting until 23 to 28 days (prealbumin) or 41 to 60 days (transferrin) after injury $(P<0.05)$ (Fig. 3F-G) Both apolipoprotein A1 and apolipoprotein B were also lower in nonsurvivors than in survivors. Apolipoprotein A1 was slightly lower from 11 to 16 days until 61 to 90 days after burn injury $(P<0.05)$ (Fig. 3I), whereas apolipoprotein B was lower from 11 to 16 days to

\begin{tabular}{|c|c|c|c|}
\hline Characteristic & Nonsurvivor $(n=79)$ & Survivor $(n=151)$ & $P$ \\
\hline Mortality, n (\%) & $79(100)$ & $0(0)$ & $<0.001$ \\
\hline \multicolumn{4}{|l|}{ Gender } \\
\hline Male & 45 & 85 & \\
\hline Female & 34 & 64 & \\
\hline Age at admission (yrs) & $8 \pm 6$ & $7 \pm 5$ & NS \\
\hline Inhalation injury, n (\%) & $51(65)$ & $65(43)$ & \\
\hline \multicolumn{4}{|l|}{ Type of burn } \\
\hline Flame, n (\%) & $68(86)$ & $123(82)$ & \\
\hline Scald, n (\%) & $9(11)$ & $23(15)$ & \\
\hline Other, n (\%) & $2(3)$ & $5(3)$ & \\
\hline TBSA burned (\%) & $74 \pm 19$ & $70 \pm 19$ & NS \\
\hline TBSA with third-degree burns (\%) & $65 \pm 26$ & $60 \pm 27$ & NS \\
\hline Time from burn to admission (d) & $2.9 \pm 3.5$ & $2.9 \pm 6.5$ & NS \\
\hline No. operations & $5.8 \pm 4.3$ & $5.9 \pm 4.3$ & NS \\
\hline LOS ICU (d) & $35 \pm 39$ & $45 \pm 34$ & NS \\
\hline LOS/TBSA & $0.5 \pm 0.4$ & $0.6 \pm 0.4$ & $<0.005$ \\
\hline Max Denver 2 score & $6.7 \pm 2.2$ & $3.4 \pm 1.5$ & $<0.001$ \\
\hline Number with MOF, n (\%) & $62(79)$ & $40(27)$ & $<0.001$ \\
\hline Number with sepsis, n (\%) & $43(54)$ & $11(7)$ & $<0.001$ \\
\hline No. infections & $3.4 \pm 3.1$ & $2.9 \pm 2.6$ & NS \\
\hline
\end{tabular}

Data are presented as mean \pm SD unless otherwise indicated.

ICU indicates intensive care unit; LOS, length of stay; MOF, multiple organ failure; NS, nonsignificant. 

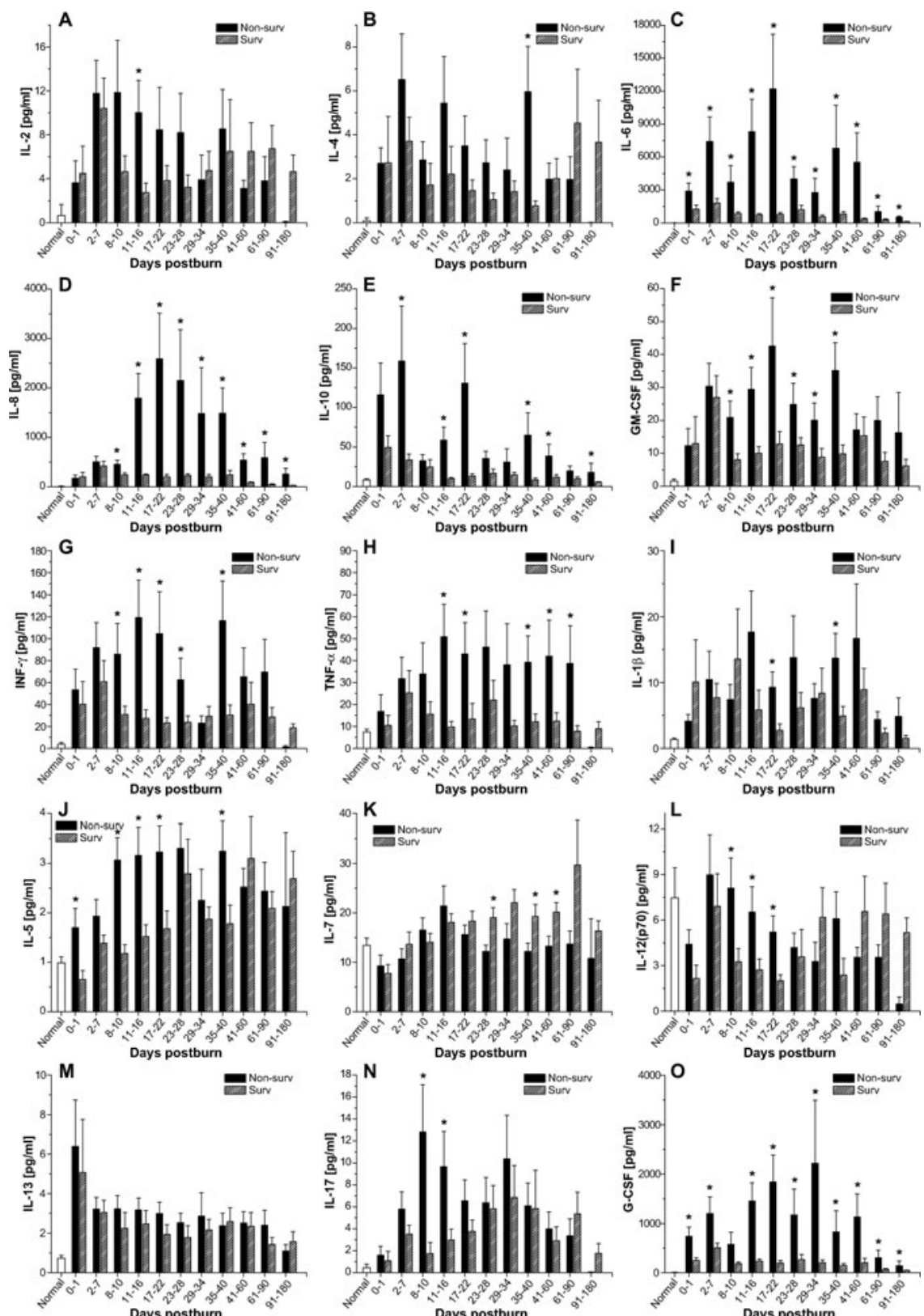

FIGURE 2. Inflammatory profiles of survivors and nonsurvivors. Serum levels of IL-2 (A), IL-4 (B), IL-6 (C), IL-8 (D), IL-10 (E), GMCSF (F), IFN- $\gamma(\mathrm{G})$, TNF- $\alpha(\mathrm{H})$, IL-1 $\beta$ (I), IL-5 (J), IL-7 (K), IL-12 (p70) (L), IL-13 (M), IL-17 $(\mathrm{N}), \mathrm{G}-\mathrm{CSF}(\mathrm{O}), \mathrm{MCP}-1$ (P), and MIP-1 $\beta(\mathrm{Q})$. Data are expressed as the mean \pm SEM. ${ }^{*} P$ $<0.05$ for survivors vs nonsurvivors.
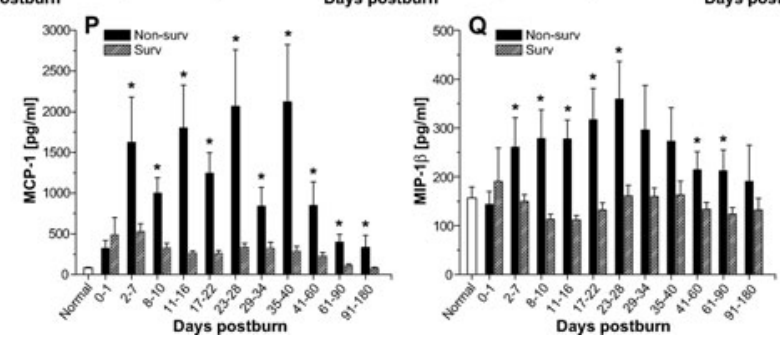

23 to 28 days after injury $(P<0.05)$ (Fig. 3J). Serum triglycerides gradually increased upon burn injury and were significantly elevated levels between 17 and 180 days posttrauma (Fig. 3K).

Serum glucose levels were significantly higher in nonsurvivors than in survivors. This difference was apparent 2 to 7 days after burn injury and lasted 61 to 90 days after injury $(P<0.05)$ (Fig. 4A). Serum glucose significantly increased immediately upon burn injury to levels of $156 \pm 2 \mathrm{mg} / \mathrm{dL}$ and remained significantly elevated for a period of 180 days before gradually decreasing to levels within the normal physiologic range, $P<0.05$. Soon after injury, serum insulin 

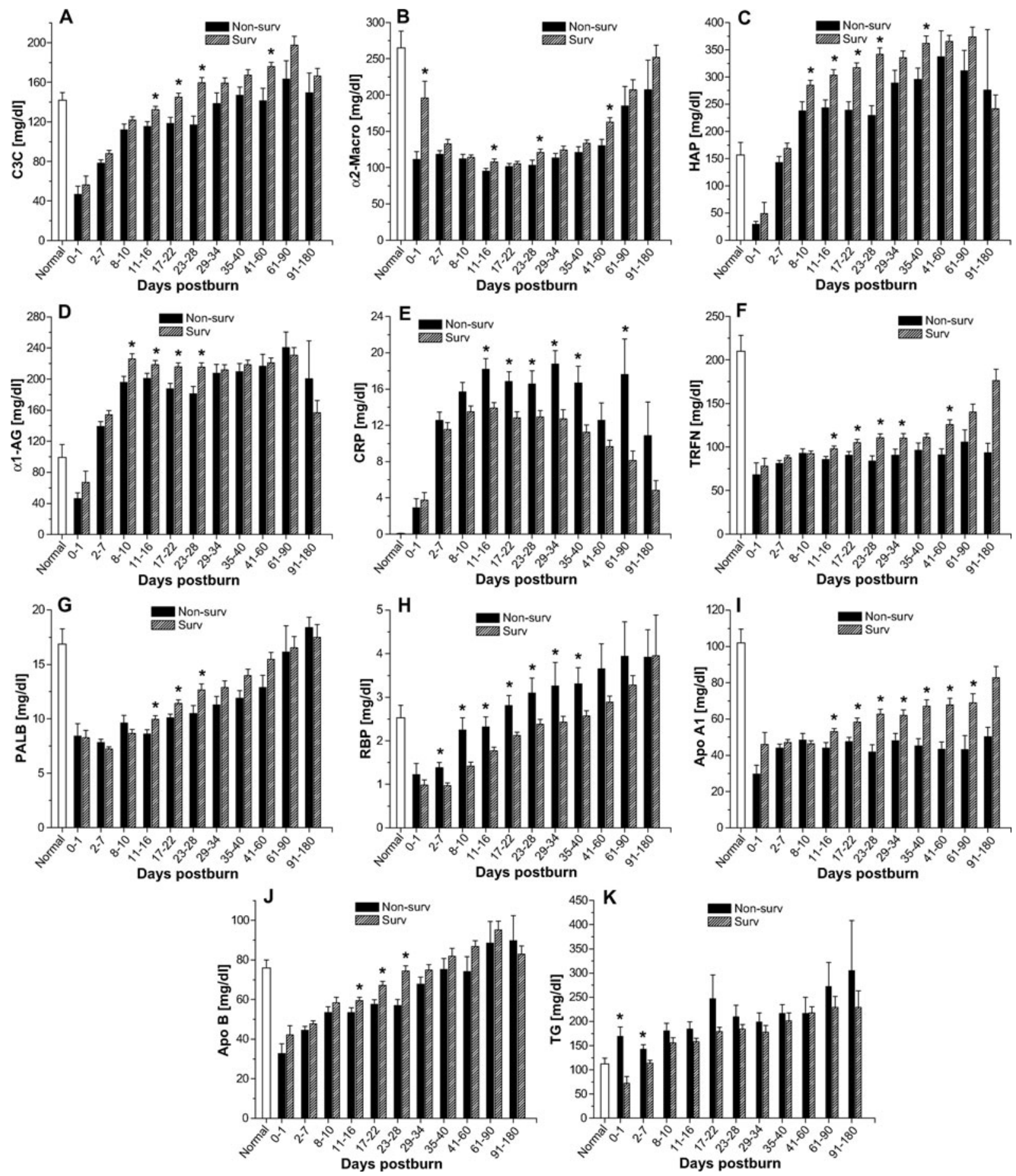

FIGURE 3. Hepatic protein levels in survivors and nonsurvivors. Serum levels of complement C3 (A), $\alpha 2$-macroglobulin $(B)$, haptoglobin $(C)$, $\alpha 1$-acid glycoprotein $(D), C$-reactive protein $(E)$, transferrin $(F)$, prealbumin $(G)$, retinol-binding protein $(\mathrm{H})$, apolipoprotein A1 (I), apolipoprotein B (I), and triglycerides (K). Data are expressed as the mean \pm SEM. ${ }^{* P}<0.05$ for survivors vs nonsurvivors. 
FIGURE 4. Glucose and insulin levels in survivors and nonsurvivors. Serum levels of glucose $(A)$ and insulin (B). Data are expressed as the mean \pm SEM. ${ }^{*} P<0.05$ for survivors vs nonsurvivors.
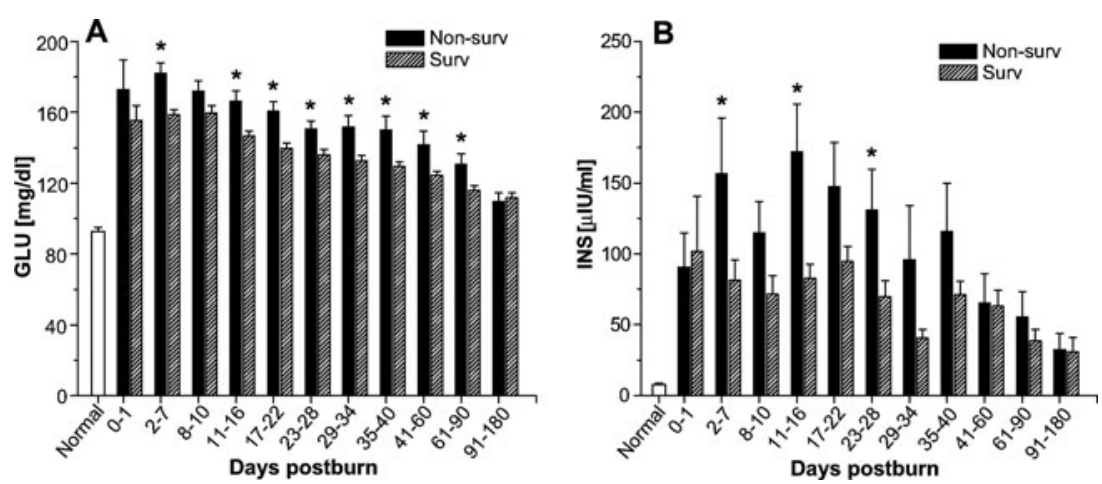

levels were also higher in nonsurvivors and remained elevated for 23 to 28 days $(P<0.05)$ (Fig. 4B).

Alanine aminotransferase concentrations were almost twofold higher in nonsurvivors than in survivors at the initial time point $(0-1$ day postburn) $(P<0.05)$ (Fig. 5A). A similar increase in aspartate aminotransferase was noted in nonsurvivors at 8 to 10 days postburn $(P<0.05)$. More moderate increases in aspartate aminotransferase were also present from 17 to 22 days to 41 to 60 days $(P<$ 0.05) (Fig. 5B). Despite supplementation of albumin, we found that serum albumin levels were significantly lower in nonsurvivors than those seen in survivors at 3 time points in the 8- to 34-day period $(P<0.05)$ (Fig. 5C). Alkaline phosphatase levels were moderately lower in nonsurvivors 11 to 22 days after burn injury $(P<0.05)$ (Fig. 5D). Finally, blood urea nitrogen, creatinine, and total bilirubin levels were two- to sevenfold higher in nonsurvivors than in survivors $(P<0.05)$ (Fig. 5E-G). These increased levels were present at almost all time points examined.

\section{Serum Hormones}

Of the 11 serum hormones measured, 7 differed between nonsurvivors and survivors (Fig. 8). Of these, only parathyroid hormone was found to be lower in nonsurvivors than in survivors $(P<0.05)$ (Fig. 8). As already mentioned, insulin was elevated in nonsurvivors relative to survivors. Other hormones that were elevated included insulin-like growth factor binding protein-3, human growth hormone, osteocalcin, and estrogen $(P<0.05$ vs survivors $)$. These increases occurred within 34 days postburn.

\section{Hypermetabolism and Cardiac Function}

As expected, burn patients exhibited hypermetabolism, as seen by an increase in the predicted REE at admission and an even greater increase at discharge. ${ }^{16,17}$ Analysis of the differences between nonsurvivors and survivors revealed that predicted REE was approximately $10 \%$ greater in the former group at discharge $(P<0.05)$ (Fig. 6). Because burn-induced hypermetabolism is accompanied by impaired cardiac function, we also compared cardiac function between nonsurvivors and survivors. We found that nonsurvivors had approximately $40 \%$ higher $\mathrm{CO}$ at 29 to 34 days and 91 to 180 days $(P<0.05)$ (Fig. 7A), approximately $30 \%$ higher CI at 29 to 34 days $(P<0.05)$ (Fig. 7B), and approximately $40 \%$ higher SV at 91 to 180 days $(P<$ 0.05 ) (Fig. 7C). In contrast, nonsurvivors had a lower heart rate than survivors at 61 to 90 days postburn $(P<0.05)$ (Fig. 7D).

\section{DISCUSSION}

Advances in therapeutic strategies, based on improved understanding of resuscitation, enhanced wound coverage, more appropriate infection control, improved treatment of inhalation injury, standardized critical care, and better support of the hypermetabolic response to injury have improved the clinical outcome of severely burned patients in recent years. ${ }^{1-3}$ However, severe burns remain a devastating injury affecting nearly every organ system and leading to significant morbidity and mortality. To obtain information about the patient's survivability and subsequent personalization of the patients care, we set out to determine whether we could identify trajectories in patients who survived a burn injury and compare these trajectories to patients who did not survive a similar size burn injury. We found that patients who succumbed to the injury had in fact profound differences in inflammatory, acute phase, and metabolic responses.

Cytokines as part of the inflammatory response are considered to be particularly important mediators of the pathophysiological process postburn, because severe burn trauma is known to induce a distinct systemic inflammatory reaction in patients. ${ }^{14}$ The postburn release of proinflammatory mediators is associated with the induction of the hypermetabolic response and with protein wasting and organ dysfunction, ${ }^{4,7}$ contributing to increased incidences of infection and sepsis, factors that augment the risk of multiple organ failure and death. ${ }^{14}$ Early markers of burn-induced inflammation include IL$1 \beta$, IL-6, IL- 8 , IL-10, and TNF- $\alpha$. In severely burned patients, IL-1 and IL- 8 peak at the time of patient's admission to the hospital and therefore represent key markers to distinguish between survivors and nonsurvivors.

IL-1 is a critical component of the inflammatory mediator cascade, regulating the host response to infection, injury, and inflammation. ${ }^{18} \mathrm{IL}-1$ is especially critical for the maintenance of a persistent inflammatory state in the lungs of patients with acute respiratory distress syndrome. ${ }^{19}$ Similarly to IL-8, IL-6 plays a pivotal role in mediating the acute phase response. However, prolonged and excessive elevations of circulating IL- 6 and IL- 8 levels in patients after trauma, severe burn, and elective surgery have been highly associated with complications and mortality. In our study, we found dramatic changes in serum IL-6, IL-8, G-CSF, and MCP-1 in the nonsurvivor group. Although IL- 6 and IL- 8 have been extensively studied and have been correlated with total burn surface area, severity of skin burn injury and survival, G-CSF, and MCP-1 have been less discussed in this context. MCP- 1 is a member of the $\beta$-chemokines and plays a crucial role in the trafficking and recruitment of effector leukocytes to primary sites of immune responses and inflammation..$^{20,21}$ Recent reports demonstrate that MCP-1 induces insulin resistance in adipocytes and skeletal muscle cells. ${ }^{22}$ Inflammatory cytokines such as TNF, IL-6, and MCP-1 have been also shown to inhibit insulin action through modification of signaling properties of insulin-receptor substrates, contributing to liver and skeletal muscle insulin resistance ${ }^{23,24}$ and hypermetabolism. In fact, we found that nonsurvivors have persistently higher metabolic rates than survivors indicating the importance of the hypermetabolic response and justifying perturbations to decrease postburn hypermetabolism. 

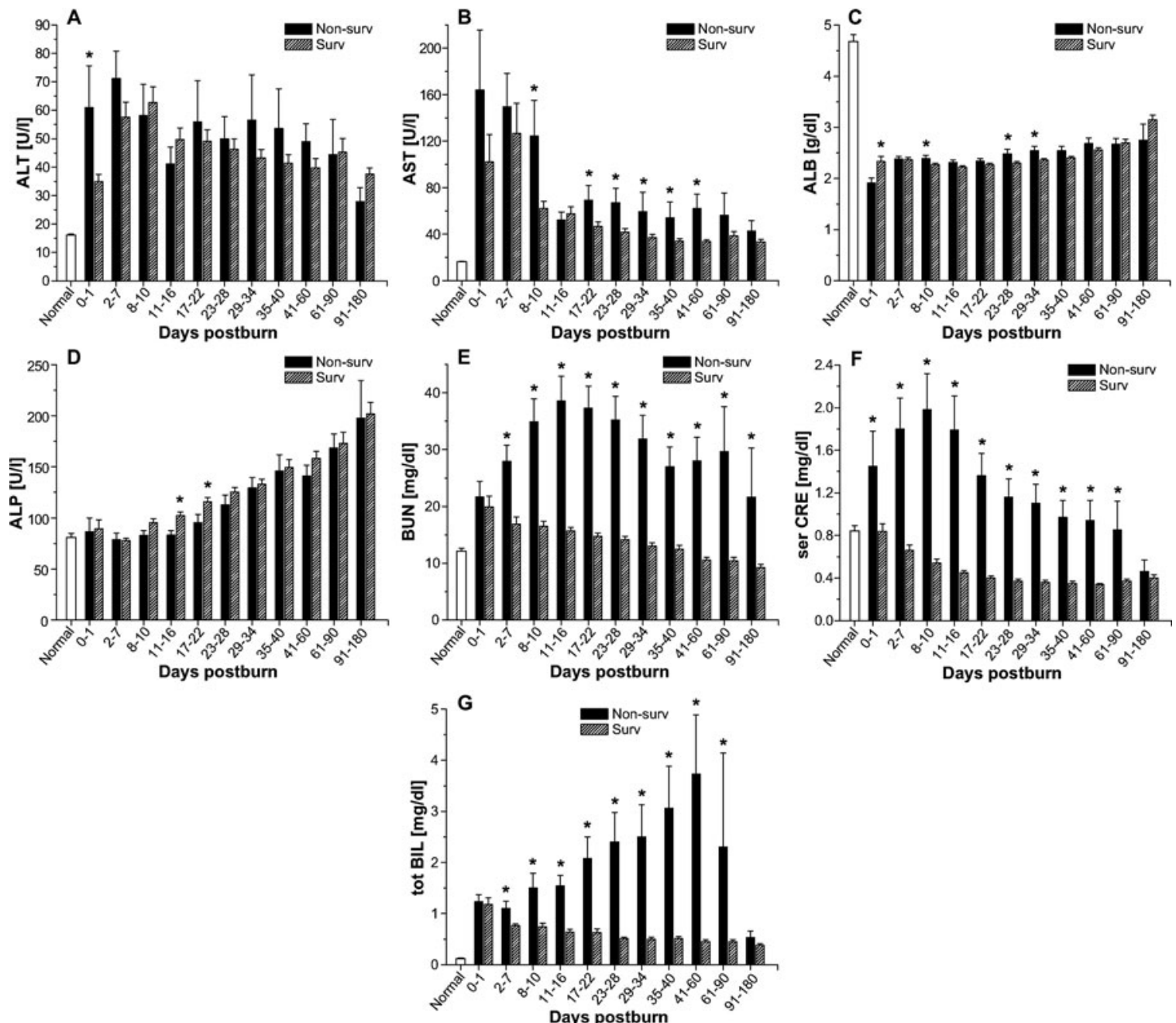

Days postburn

FIGURE 5. Common markers of organ function in survivors and nonsurvivors. Serum levels of alanine aminotransferase (A), aspartate aminotransferase (B), albumin (C), alkaline phosphatase (D), blood urea nitrogen (E), creatinine (F), and total bilirubin (G). Data are expressed as the mean \pm SEM. ${ }^{*} P<0.05$ for survivors vs nonsurvivors.

Like the inflammatory response, the acute phase response contributes to postburn morbidity and mortality, with the production of acute phase proteins and factors. In the present study, we found various significant differences between the acute phase trajectories in survivors compared with nonsurvivors, but it appears that these differences occur later during hospitalization and not early on, decreasing the value of acute phase protein as predictors of mortality. One of the few interesting biomarkers was serum triglycerides. It seems that triglycerides are significantly elevated in nonsurvivors during the early phase postburn and hence represent a potential effective biomarker, confirming a previous study. ${ }^{25}$

Insulin resistance and hyperglycemia have been associated with poor outcome postburn ${ }^{8,26-28}$ and we have recently shown in a randomized controlled trial in severely burned children that tight glucose control improves postburn outcomes. ${ }^{9}$ It is not entirely clear by which mechanisms insulin resistance and hyperglycemia cause worsened outcomes, but it appears that both cause protein glycosylation, increased free radicals, impaired wound healing, increased skin graft loss, increased muscle protein catabolism, and increased incidence of infections and mortality. ${ }^{8,9,26,29}$ In the present study, we showed that nonsurvivors have significantly elevated glucose levels and insulin levels, indicating significantly increased insulin resistance. Increased insulin resistance was present from the initial phase postburn and hence can be used as a predictor. However, insulin resistance could be also a marker of augmented and increased hypermetabolism due to an increased illness. We hypothesize that hyperglycemia is associated with poor outcomes, and we therefore suggest glucose titration to a beneficial serum glucose target of 130 $\mathrm{mg} / \mathrm{dL} .^{30}$

Our previous studies demonstrated that the hormonal, inflammatory, and metabolic profiles vary on the basis of concomitant conditions such as presence of inhalation injury or sepsis. ${ }^{4,12,31-35}$ This study addresses the greater question of what changes occur in nonsurviving patients regardless of the cause of death or existence of concurrent injuries (such as, inhalation injuries). Further studies within our group have evaluated use of the biomarkers described here to predict patient outcome. We investigated the effect of combining proteomics variables with clinical covariates on prediction of mortality in burned children. Serum samples were collected from 330 burned children (burns covering $>25 \%$ of the TBSA) between admission 

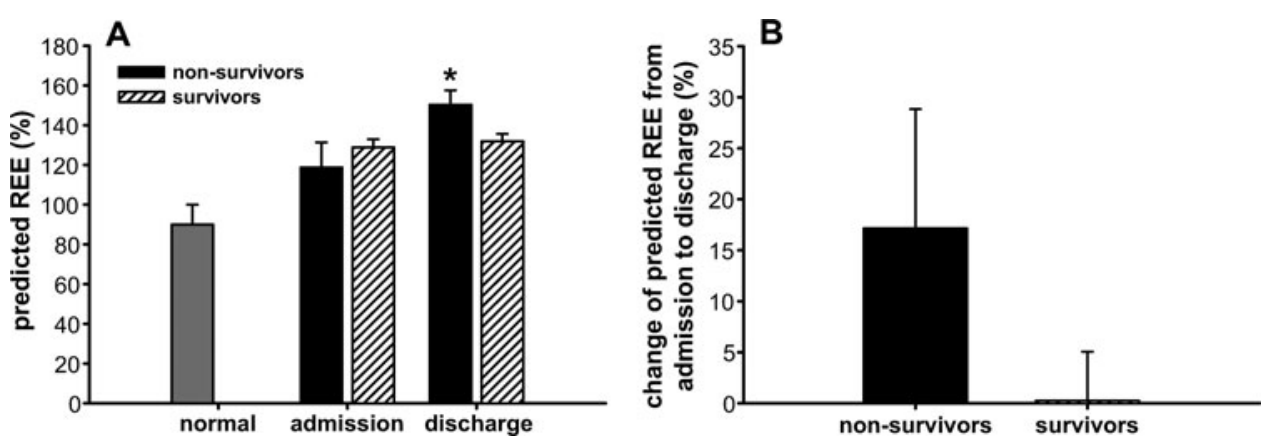

FIGURE 6. Hypermetabolism in survivors and nonsurvivors. Predicted REE (A) and change in REE from admission to discharge (B). Data are expressed as the mean \pm SEM. ${ }^{*} P<0.05$ for survivors vs nonsurvivors. REE indicates resting energy expenditure.
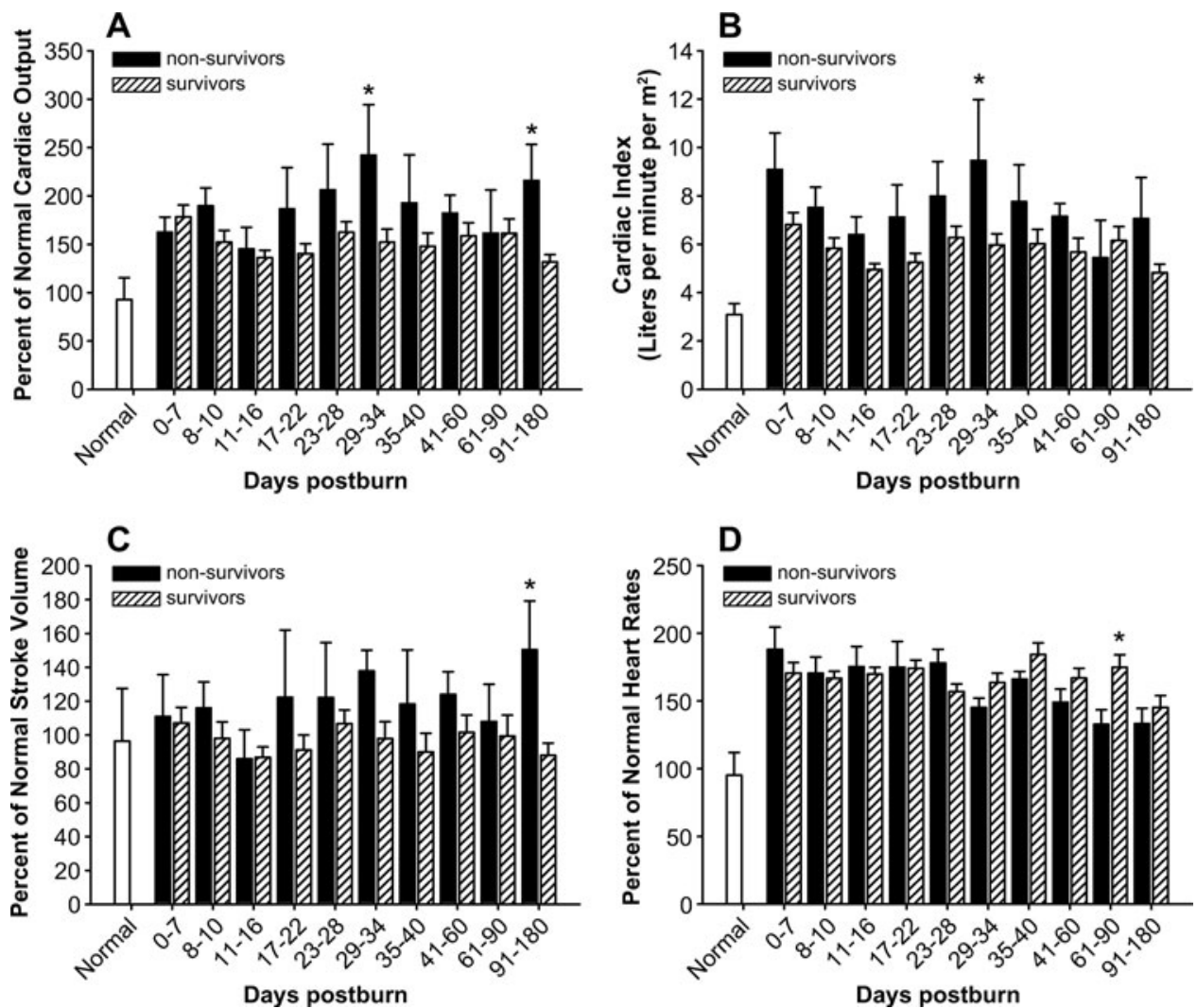

FIGURE 7. Cardiac function in survivors and nonsurvivors. Cardiac output (A), cardiac index (B), stroke volume (C), and heart rate (D). Data are expressed as the mean \pm SEM. ${ }^{*} P<0.05$ for survivors vs nonsurvivors.

and the time of the first operation for clinical chemistry analyses and proteomic assays of cytokines. Principal component analysis revealed that serum protein abundance and the clinical covariates each provided independent information regarding patient survival. To determine whether combining proteomics with clinical variables improves prediction of patient mortality, we used multivariate adaptive regression splines, because the relationships between analytes and mortality were not linear. Combining these factors increased overall outcome prediction accuracy from $52 \%$ to $81 \%$ and area under the receiver operating characteristic curve from 0.82 to 0.95 . Thus, the predictive accuracy of burns mortality is substantially improved by combining protein abundance information with clinical covariates in a multivariate adaptive regression splines classifier. Despite the weakness of a single time-point model, this study indicates the importance and feasibility of constructing models to predict burn outcomes. ${ }^{10}$

The temporal alterations in concentrations of inflammatory, hormonal, and acute phase response proteins that occur in nonsurvivors demonstrate the persistence of the immune dysfunction, loss of insulin sensitivity, and hepatic impairment that occur after a severe burn injury. Furthermore, the differences in survivors and nonsurvivors appear to be in the extent and duration of the response. Despite apparent decreases in analyte concentrations in surviving patients, these values are still significantly elevated above concentrations in nonburned patients; the same is true for apparent increases in these biomarkers. That the inflammatory and metabolic responses are magnified is a theme that we also see repeated in alterations within 


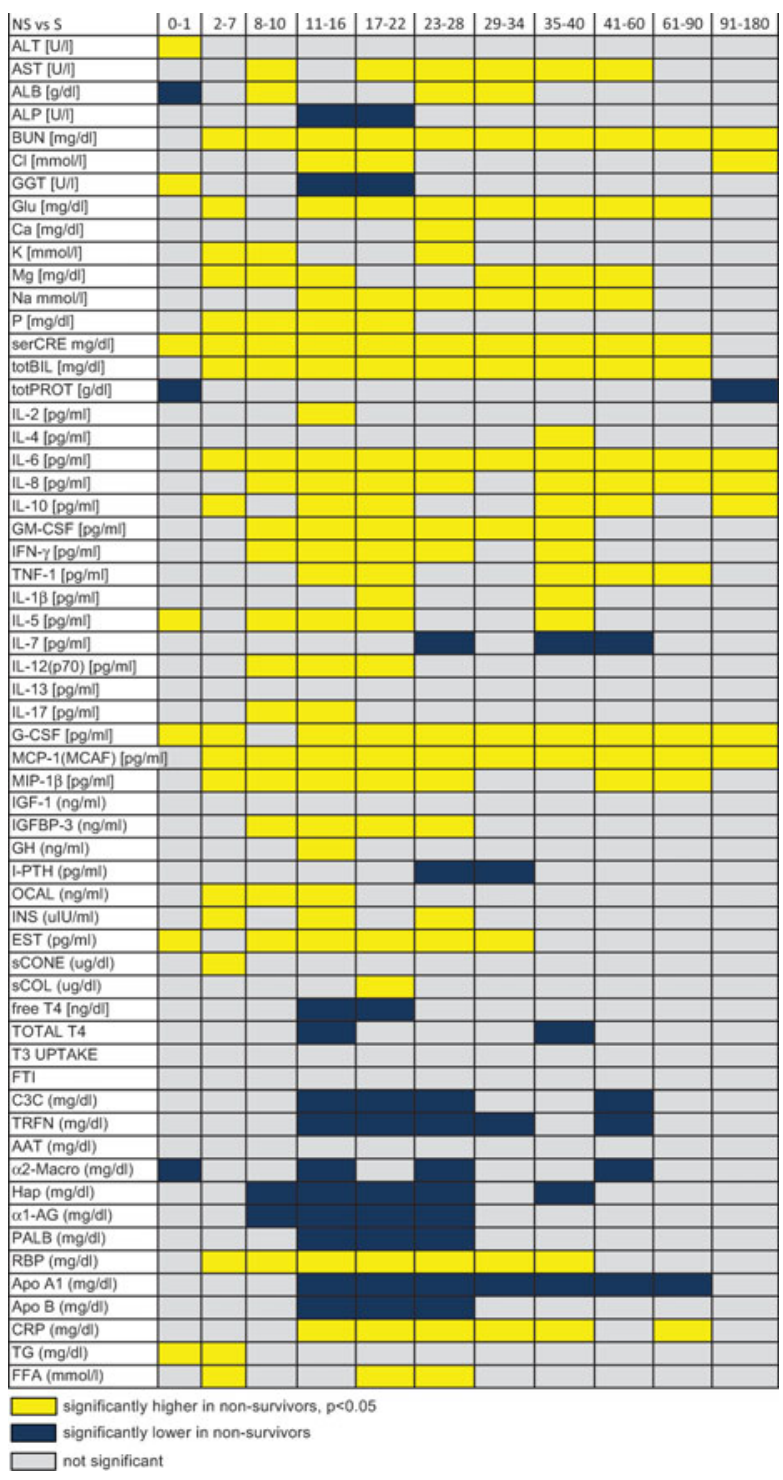

FIGURE 8. Comparison of serum profiles for cytokines, hormones, and other factors in survivors and nonsurvivors.

the genomic expression profiles of peripheral blood leukocytes (unpublished observations). Although we can only speculate with respect to the mechanistic implications of the alterations or the extreme nature of the responses in nonsurvivors, the utility of these analytes as biomarkers for survival is clear. We have identified, for the first time, specific temporally expressed markers that can be used to identify patients with a high likelihood of nonsurvival at discrete time points. This knowledge can be used to monitor patient progress and to identify, in a prospective manner, patients who may need additional interventions to improve their chances of survival. It is now desirable to confirm these trajectories prospectively in a new population of burn patients and to determine whether adults and elderly people have also trajectories that differentiate survivors from nonsurvivors.

\section{ACKNOWLEDGMENTS}

The authors thank the following research staff for their help and assistance: Deb Benjamin, Wes Benjamin, Joanna Huddleston,
Lucy Robles, Sylvia Ojeda, Rosa Chapa, Guadalupe Jecker, Mary Kelly, Karen Henderson, Maria Magno, Liz Montemayor, Kristin Rojas, Victor Baras, and Maricela Pantoja. They extend special thanks to Eileen Figueroa, Steven Schuenke, and Dr. Kasie Cole for their assistance in manuscript preparation. The author contributions were as follows: M.G.J. did patient care, collected the data, developed study design, and wrote the manuscript; D.N.H. did patient care, developed the study design, collected data, and edited the manuscript; C.C.F. collected data, did statistical analysis, and edited the manuscript; R.K. did the statistical analysis and edited the manuscript; R.P.M. collected and edited the manuscript; G.G.G. did the study design, collected data, did statistical analysis, and wrote the manuscript.

\section{REFERENCES}

1. Herndon DN, Tompkins RG. Support of the metabolic response to burn injury. Lancet. 2004;363:1895-1902.

2. Kraft R, Herndon DN, Al-Mousawi AM, et al. Burn size and survival probability in paediatric patients in modern burn care: a prospective observational cohort study. Lancet. 2012;379:1013-1021.

3. Williams FN, Herndon DN, Hawkins HK, et al. The leading causes of death after burn injury in a single pediatric burn center. Crit Care. 2009;13:R183

4. Jeschke MG, Chinkes DL, Finnerty CC, et al. Pathophysiologic response to severe burn injury. Ann Surg. 2008;248:387-401.

5. Reiss E, Pearson E, Artz CP. The metabolic response to burns. J Clin Invest 1956;35:62-77.

6. Gauglitz GG, Herndon DN, Kulp GA, et al. Abnormal insulin sensitivity persists up to three years in pediatric patients post-burn. J Clin Endocrinol Metab. 2009;94:1656-1664.

7. Jeschke MG, Gauglitz GG, Kulp GA, et al. Long-term persistence of the pathophysiologic response to severe burn injury. PLoS One. 2011;6:e21245.

8. Gore DC, Chinkes D, Heggers J, et al. Association of hyperglycemia with increased mortality after severe burn injury. J Trauma. 2001;51:540-544.

9. Jeschke MG, Kulp GA, Kraft R, et al. Intensive insulin therapy in severely burned pediatric patients: a prospective randomized trial. Am J Respir Crit Care Med. 2010;182:351-359.

10. Finnerty CC, Ju H, Spratt H, et al. Proteomics improves the prediction of burns mortality: results from regression spline modeling. Clin Transl Sci. 2012;5:243-249.

11. Finnerty CC, Herndon DN, Jeschke MG. Inhalation injury in severely burned children does not augment the systemic inflammatory response. Crit Care. 2007;11:R22.

12. Gauglitz GG, Finnerty CC, Herndon DN, et al. Are serum cytokines early predictors for the outcome of burn patients with inhalation injuries who do not survive? Crit Care. 2008;12:R81

13. Greenhalgh DG, Saffle JR, Holmes JH, IV, et al. American Burn Association consensus conference to define sepsis and infection in burns. J Burn Care Res. 2007;28:776-790.

14. Finnerty CC, Herndon DN, Przkora R, et al. Cytokine expression profile over time in severely burned pediatric patients. Shock. 2006;26:13-19.

15. Mlcak RP, Jeschke MG, Barrow RE, et al. The influence of age and gender on resting energy expenditure in severely burned children. Ann Surg. 2006;244:121-130.

16. Jeschke MG, Mlcak RP, Finnerty CC, et al. Burn size determines the inflammatory and hypermetabolic response. Crit Care. 2007;11:R90.

17. Williams FN, Herndon DN, Suman OE, et al. Changes in cardiac physiology after severe burn injury. J Burn Care Res. 2011;32:269-274.

18. Kaplan E, Dinarello CA, Gelfand JA. Interleukin-1 and the response to injury. Immunol Res. 1989;8:118-129.

19. Goodman RB, Pugin J, Lee JS, et al. Cytokine-mediated inflammation in acute lung injury. Cytokine Growth Factor Rev. 2003;14:523-535.

20. Furukawa K, Kobayashi M, Herndon DN, et al. Appearance of monocyte chemoattractant protein 1 (MCP-1) early after thermal injury: role in the subsequent development of burn-associated type 2 T-cell responses. Ann Surg. 2002;236:112-119.

21. Luster AD. Chemokines - chemotactic cytokines that mediate inflammation N Engl J Med. 1998;338:436-445.

22. Sell H, Eckel J. Monocyte chemotactic protein-1 and its role in insulin resistance. Curr Opin Lipidol. 2007;18:258-262. 
23. del Aguila LF, Claffey KP, Kirwan JP. TNF-alpha impairs insulin signaling and insulin stimulation of glucose uptake in $\mathrm{C} 2 \mathrm{C} 12$ muscle cells. Am J Physiol. 1999;276:E849-855.

24. Fan J, Li YH, Wojnar MM, et al. Endotoxin-induced alterations in insulinstimulated phosphorylation of insulin receptor, IRS-1, and MAP kinase in skeletal muscle. Shock. 1996;6:164-170.

25. Kamolz LP, Andel H, Mittlbock M, et al. Serum cholesterol and triglycerides: potential role in mortality prediction. Burns. 2003;29:810-815.

26. Gore DC, Chinkes DL, Hart DW, et al. Hyperglycemia exacerbates muscle protein catabolism in burn-injured patients. Crit Care Med. 2002;30:24382442.

27. Hemmila MR, Taddonio MA, Arbabi S, et al. Intensive insulin therapy is associated with reduced infectious complications in burn patients. Surgery. 2008;144:629-635; discussion 635-637.

28. Pham TN, Warren AJ, Phan HH, et al. Impact of tight glycemic control in severely burned children. J Trauma. 2005;59:1148-1154.
29. Jeschke MG, Klein D, Herndon DN. Insulin treatment improves the systemic inflammatory reaction to severe trauma. Ann Surg. 2004;239:553-560.

30. Jeschke MG, Kraft R, Emdad F, et al. Glucose control in severely thermally injured pediatric patients: what glucose range should be the target? Ann Surg. 2010;252:521-527; discussion 527-528.

31. Finnerty CC, Herndon DN, Chinkes DL, et al. Serum cytokine differences in severely burned children with and without sepsis. Shock. 2007;27:4-9.

32. Gauglitz GG, Toliver-Kinsky TE, Williams FN, et al. Insulin increases resistance to burn wound infection-associated sepsis. Crit Care Med. 2010;38:202208.

33. Hart DW, Wolf SE, Chinkes DL, et al. Determinants of skeletal muscle catabolism after severe burn. Ann Surg. 2000;232:455-465.

34. Hart DW, Wolf SE, Mlcak R, et al. Persistence of muscle catabolism after severe burn. Surgery. 2000;128:312-319.

35. Jeschke MG, Micak RP, Finnerty CC, et al. Changes in liver function and size after a severe thermal injury. Shock. 2007;28:172-177. 